\title{
Stimulation of airway and intestinal mucosal secretion by natural coumarin CFTR activators
}

\author{
Hong Yang ${ }^{1}$, Li-Na Xu ${ }^{2}$, Yu-jie Sui ${ }^{2}$, Xin Liu ${ }^{3}$, Cheng-yan He ${ }^{3}$, Rou-yu Fang ${ }^{2}$, Jia Liu ${ }^{2}$, Feng Hao and \\ Tong-Hui Ma ${ }^{1,2}$ *
}

1 School of life sciences, Liaoning Provincial Key Laboratory of Biotechnology and Drug Discovery, Liaoning Normal University, Dalian, PR China

${ }^{2}$ Central Research Laboratory, Jilin University Bethune Second Hospital, Changchun, PR China

${ }^{3}$ China-Japan Union Hospital, Jilin University, Changchun, PR China

\section{Edited by:}

Tzyh-Chang Hwang, University of

Missouri-Columbia, USA

\section{Reviewed by:}

Keliang Xie, General Hospital of

Tianjin Medical University, China

M.M. Reddy, University of California,

USA

Silvia Bompadre, University of

Missouri, USA

\section{*Correspondence:}

Hong Yang, School of Life Sciences, Liaoning Normal University, Dalian

116029, PR China.

e-mail: hyanglnnu@gmail.com

Tong-Hui Ma, Central Research

Laboratory, Jilin University Bethune

Second Hospital, Changchun 130041,

PR China.

e-mail:math108@gmail.com
Mutations of cystic fibrosis (CF) transmembrane conductance regulator (CFTR) cause lethal hereditary disease CF that involves extensive destruction and dysfunction of serous epithelium. Possible pharmacological therapy includes correction of defective intracellular processing and abnormal channel gating. In a previous study, we identified five natural coumarin potentiators of $\Delta$ F508-CFTR including osthole, imperatorin, isopsoralen, praeruptorin A, and scoparone. The present study was designed to determine the activity of these coumarine compounds on CFTR activity in animal tissues as a primary evaluation of their therapeutic potential. In the present study, we analyzed the affinity of these coumarin potentiators in activating wild-type CFTR and found that they are all potent activators. Osthole showed the highest affinity with $K_{d}$ values $<50 \mathrm{nmol} / \mathrm{L}$ as determined by Ussing chamber short-circuit current assay. Stimulation of rat colonic mucosal secretion by osthole was tested by the Ussing chamber short-circuit current assay. Osthole reached maximal activation of colonic $\mathrm{Cl}^{-}$secretion at $5 \mu \mathrm{mol} / \mathrm{L}$. Stimulation of mouse tracheal mucosal secretion was analyzed by optical measurement of single gland secretion. Fluid secretion rate of tracheal single submucosal gland stimulated by osthole at $10 \mu \mathrm{mol} / \mathrm{L}$ was three-fold more rapid than that in negative control. In both cases the stimulated secretions were fully abolished by $\mathrm{CFTR}_{\text {inh }}-172$. In conclusion, the effective stimulation of $\mathrm{Cl}^{-}$and fluid secretion in colonic and tracheal mucosa by osthole suggested the therapeutic potential of natural coumarin compounds for the treatment of CF and other CFTR-related diseases.

Keywords: CFTR, coumarins, activator, fluid secretion, mucosa, colon, airway

\section{INTRODUCTION}

Cystic fibrosis (CF) transmembrane conductance regulator (CFTR) is a multidomain glycoprotein, belongs to the adenine nucleotide-binding cassette $(\mathrm{ABC})$ transporter family (Kerem et al., 1989), and is the only anion channel of ABC superfamily (He et al., 2008). CFTR is extensively expressed on the luminal surface of serous epithelial cells most prominently in the intestines, airways, pancreas, bile ducts, epididymis, and conjunctiva (Kunzelmann, 1999; Hanrahan and Wioland, 2004; Riordan, 2008). It has been confirmed that CFTR play a vital role in the absorption and secretion of electrolytes and fluid in submucosal glands (Kunzelmann, 1999; Thiagarajah and Verkman, 2003; Riordan, 2008). In some epithelia such as in colon, CFTR may account for the entire apical chloride conductance (Greger, 2000). Besides as a cAMP-dependent chloride channel, CFTR also regulates activities of other ion channels, such as the epithelial $\mathrm{Na}^{+}$channel (ENaC; Berdiev et al., 2009), outwardly rectifying $\mathrm{Cl}^{-}$channel (ORCC; Jovov et al., 1995; Hryciw and Guggino, 2000), KvLQT1 (Boucherot et al., 2001), ROMK (Ho, 1998), and water channel (Castro-Parodi et al., 2009). In addition, CFTR may play roles in establishing the low $\mathrm{pH}$ in the biosynthetic compartments of the trans-Golgi network and in endosomes (Machen et al., 2001).
Altered function of CFTR is related to many clinical symptoms. For example, mutations in CFTR that alters its biogenesis and/or intrinsic transport function cause the hereditary lethal disease CF (Marcet and Boeynaems, 2006). On the other hand, improper activation of CFTR is involved in diseases such as secretary diarrhea (Thiagarajah and Verkman, 2003) and polycystic kidney disease (PKD; Li and Sheppard, 2009). Altered CFTR function also accounts for curtain other disorders, such as constipation (Pratha et al., 2000), keratoconjunctivitis sicca (KCS; Levin and Verkman, 2005), and idiopathic chronic pancreatitis (ICP; Cohn et al., 1998). Therefore, CFTR became a potential drug target for these diseases.

In a previous study, we identified a family of natural coumarin compounds that can potentiate $\mathrm{Cl}^{-}$transport through $\triangle \mathrm{F} 508$ CFTR by screening a collection of 386 natural single compounds from Chinese medicinal herbs (Xu et al., 2008). However, the in vivo effects of these coumarin compounds have not been determined. In the present study, we first characterized the activity of these coumarin $\triangle$ F508-CFTR potentiators on wild-type CFTR (wt-CFTR) and then examined their effect on $\mathrm{Cl}^{-}$secretion in freshly isolated rodent colonic and tracheal mucosa. The results indicated effective stimulation of $\mathrm{Cl}^{-}$secretion in live tissues and therefore support the therapeutic potential of coumarin CFTR 
potentiators for CFTR-related diseases including CF, constipation, and ICP.

\section{MATERIALS AND METHODS COUMARINS}

Coumarins were purchased from the National Institute for the Control of Pharmaceutical and Biological Products in China (purity $>99 \%$ ). The purities of compounds were confirmed by HPLC/MS analysis. Compounds were dissolved in DMSO to make a $20-\mathrm{mmol} / \mathrm{L}$ stock solution. In all tests, Cells were exposed to a final concentration of DMSO of $<1 \%$, to avoid DMSO effects on the cell-based assay.

\section{CELL LINES}

Halide-sensitive yellow fluorescent protein mutant (EYFPH148Q) and human wt-CFTR cotransfected fischer rat thyroid (FRT) epithelium were generated and cultured as references (Galietta et al., 2001; Yang et al., 2003). The cells were prepared both for fluorescence functional and electrophysiological analyses. For fluorescence assay, FRT cells were plated into a special black-walled, clear-bottomed 96-well tissue culture plate (Costar, Corning, NY, USA), and incubated at $37^{\circ} \mathrm{C}$ for $24 \mathrm{~h}$ before measurement of compound activity. For the electrophysiological Ussing chamber assay, cells were cultured on the porous supports Snapwell inserts (Costar, Corning, NY, USA) at $37^{\circ} \mathrm{C}$ for 7 days before measurements.

\section{FLUORESCENCE ASSAY OF CFTR CHANNEL FUNCTION}

Fischer rat thyroid cells grown in 96-well tissue culture plates were washed three times with phosphate-buffered saline (PBS), then incubated with $100 \mathrm{nmol} / \mathrm{L}$ forskolin without (control) or with coumarin in a final volume of $40 \mu \mathrm{l}$ for $10 \mathrm{~min}$. The fluorescence of each well was recorded for $14 \mathrm{~s}$ (five points per second) continuously in a fluorescence plate reader (FLUOstar Optima; BMG Laboratory Technologies, Offenburg, Germany) with $2 \mathrm{~s}$ before and $12 \mathrm{~s}$ after injection of $120 \mu \mathrm{l}$ of and $\mathrm{I}^{-}$-containing solution (PBS with $137 \mathrm{mM} \mathrm{Cl}^{-}$replaced by equal concentration of $\mathrm{I}^{-}$). $\mathrm{I}^{-}$ influx rates $\left(\mathrm{d}\left[\mathrm{I}^{-}\right] / \mathrm{dt}\right.$ at $\left.t=0\right)$ were computed from fluorescence time course data by single exponential regression, as described previously (Ma et al., 2002).

\section{ELECTROPHYSIOLOGY}

Transepithelial $\mathrm{Cl}^{-}$current were measured on both CFTR transfected FRT cells and rat mucosa. FRT cells were cultured on Snapwell inserts, grown at an air-liquid interface, and placed in a Ussing chamber system (Vertical Diffusion Chamber, Physiological Instruments, San Diego, CA, USA). Measurements were performed in the presence of a transepithelial $\mathrm{Cl}^{-}$gradient, in which the basolateral side solution contained (in mmol/L): 130 $\mathrm{NaCl}, 2.7 \mathrm{KCl}, 1.5 \mathrm{KH}_{2} \mathrm{PO}_{4}, 1 \mathrm{CaCl}_{2}, 0.5 \mathrm{MgCl}_{2}, 10 \mathrm{Na}-\mathrm{HEPES}$, $\mathrm{pH} 7.3$, and 10 glucose; The apical side solution contained the same components except that $65 \mathrm{mmol} / \mathrm{L} \mathrm{NaCl}$ was replaced by sodium gluconate and the concentration of $\mathrm{CaCl}_{2}$ was increased to $2 \mathrm{mmol} / \mathrm{L}$. The basolateral membrane was permeabilized with $250 \mu \mathrm{g} / \mathrm{ml}$ amphotericin B (He et al., 2005).

For measurements of transepithelial $\mathrm{Cl}^{-}$current on rat colonic mucosa, rat colonic mucosa was obtained as described previously (He et al., 2005). Briefly, SD rats (weight about $200 \mathrm{~g}$ ) were starved for $24 \mathrm{~h}$ prior. Segments of colon were excised after the rat was sacrificed with intravenous pentobarbital $(100 \mathrm{mg} / \mathrm{kg})$. The colon was immediately stripped of muscularis, mounted in Ussing chambers (area $1.03 \mathrm{~cm}^{2}$ ), and bathed in Krebs-Henseleit (KH) solution (in mmol/L: $\mathrm{NaCl} 117, \mathrm{KCl} 4.7, \mathrm{MgCl}_{2} 1.2, \mathrm{KH}_{2} \mathrm{PO}_{4}$ 1.2, $\mathrm{NaHCO}_{3}$ 24.8, $\mathrm{CaCl}_{2}$ 2.5, glucose 11.1, pH 7.4). Shortcircuit current was measured after inhibition of $\mathrm{Na}^{+}$current by amiloride $(10 \mu \mathrm{mol} / \mathrm{L})$ and prostaglandin generation inhibitor of indomethacin $(10 \mu \mathrm{mol} / \mathrm{L})$, followed by stimulation by forskolin $(20 \mu \mathrm{mol} / \mathrm{L})$ and subsequent inhibitor addition (Ma et al., 2002). All in vivo studies including isolation of rat colonic mucosa and mouse trachea followed guidelines for animal experiments in Jilin University.

Measurements were performed at $37^{\circ} \mathrm{C}$, and solutions were continuously bubbled with air. Short-Circuit current was recorded with a DVC-1000 voltage clamp (World Precision Instruments, Sarasota, FL, USA) via $\mathrm{Ag} / \mathrm{AgCl}$ electrodes and $1 \mathrm{~mol} / \mathrm{L} \mathrm{KCl}$ agar bridges.

\section{MEASUREMENT OF INDIVIDUAL SUBMUCOSAL GLAND FLUID SECRETION}

Freshly excised the most cranial part of mouse trachea was immediately mounted on a sponge. The system was soaked in $\mathrm{KH}$ solution and held in a perfusion chamber with the mucosal side up in a humidified $5 \% \mathrm{CO}_{2} / 95 \%$ air atmosphere at $37^{\circ} \mathrm{C}$ during tests as described previously (Song and Verkman, 2001). After the mucosa was cleared with saline and dried by nitrogen stream, $200 \mu \mathrm{L}$ saline saturated oil was added to the surface. In some experiments the tissues were incubated for $30 \mathrm{~min}$ in $\mathrm{KH}$ buffer containing $20 \mu \mathrm{mol} / \mathrm{L}$ $\mathrm{CFTR}_{\text {inh }}$-172. Gland fluid droplets were imaged and recorded by a reflected light microscopy (Olympus, Olympus Micro DP Controller) after stimulation with compounds. The rate of fluid secretion from individual submucosal gland was computed from fluid droplet diameter assuming semi-spherical droplet geometry as reference (Song and Verkman, 2001).

\section{STATISTICAL ANALYSIS}

Data are reported as means \pm SE. Statistical significance of the effects was determined by using the OriginPro 8.0 software.

\section{RESULTS}

Initial experiments were preformed to verify activation of wtCFTR by five coumarin $\triangle$ F508-CFTR potentiators we identified previously (Xu et al., 2008). The five coumarins (structures shown in Figure 1A) stimulated wt-CFTR-mediated $\mathrm{I}^{-}$influx in a concentration-dependent way, with an affinity sequence from higher to lower as: osthole $>$ scoparone $>$ imperatorin $>$ isopsoralen $>$ praeruptorin A (Figure 1B). The potency and efficacy of osthole are much higher than the known CFTR potentiator genistein.

CFTR activation potencies of coumarins were further analyzed on the more reliable short-circuit current assay. Measurements were performed after the basolateral membrane of FRT cells were permeabilized with amphotericin B and in the presence of transepithelial $\mathrm{Cl}^{-}$gradient. As the representative curves shown in Figure 2A and summarized in Figure 2B, the three potent coumarin activators identified in the fluorescent 
assay stimulated transepithelial $\mathrm{Cl}^{-}$current in the presence of $0.1 \mu \mathrm{mol} / \mathrm{L}$ FSK. Noticeably, osthole exhibited very high affinity (with $K_{\mathrm{d}}<50 \mathrm{nmol} / \mathrm{L}$ ). The CFTR specific blocker CFTR $_{\text {inh }}-172$ at $20 \mu \mathrm{mol} / \mathrm{L}$ completely blocked the activations. These data indicated that the coumarin compounds stimulated $\mathrm{Cl}^{-}$currents through CFTR.

Osthole was used to further evaluate the potential effects of coumarin compounds on CFTR-mediated $\mathrm{Cl}^{-}$secretion in live tissues. The first set of experiments was performed in strips of

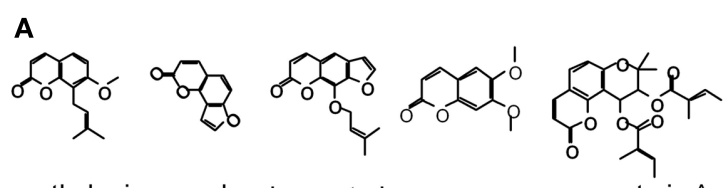

osthole isopsoralen imperatorin scoparone praeruptorin A

\section{B}

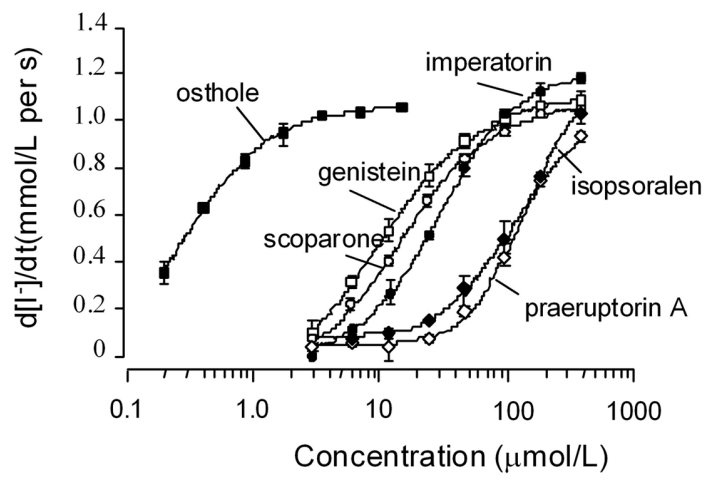

FIGURE 1 | Functional analysis of natural coumarin compounds in FRT cells expressing wild-type CFTR. (A) Chemical structures of the active coumarins. (B) Concentration-response curves for the active coumarins. Assays were performed in the presence of $0.1 \mu \mathrm{mol} / \mathrm{L}$ forskolin. Genistein was used as a positive control. (mean $\pm \mathrm{SE}, n=4$ ). rat distal colonic mucosa by short-circuit current assays. Osthole was applied on mucosal (Figure 3A) and serosal (Figure 3B) sides separately. Maximal stimulations were obtained at osthole concentration of $5 \mu \mathrm{mol} / \mathrm{L}$. CFTR inh -172 completely abolished the osthole-stimulated chloride secretion. Quantified effects of osthole on rat colonic mucosa $\mathrm{Cl}^{-}$secretion were shown in Figure 3C.

Another set of experiments was performed in freshly excised fragments of the cranial portion of mouse trachea. The effect of osthole on the rates of fluid secretion from airway submucosal glands was measured from the growth of individual fluid droplets visualized by low-magnification light microscopy. Gland fluid secretion was stimulated by addition of $10 \mu \mathrm{mol} / \mathrm{L}$ pilocarpine or $10 \mu \mathrm{mol} / \mathrm{L}$ osthole to the serosal bathing solution. Figure 4A shows a series of images of expanding fluid droplets secreted by single submucosal glands after stimulation by osthole, with pilocarpine as positive control. Figure $4 \mathrm{~B}$ summarizes the fluid secretion rate of individual submucosal gland stimulated by the indicated maneuvers. Osthole increased fluid secretion rate by 3.34-fold compared to the saline control. The effect was largely abolished by CFTR $_{\text {inh }}-172$.

\section{DISCUSSION}

In a previous study, we identified a family of natural coumarin compounds (including osthole, scoparone, imperatorin, praeruptorin $\mathrm{A}$, and isopsoralen) that can rescue defective $\triangle \mathrm{F} 508$-CFTR chloride channel gating (Xu et al., 2008). The present study was designed to determine the activity of these coumarin compounds on CFTR activity in animal tissues as a primary evaluation of their therapeutic potential.

We first confirmed the activity of the five coumarin $\Delta$ F508CFTR potentiators on wt-CFTR and identified osthole as a low nonamolar affinity activator $\left(K_{\mathrm{d}}<50 \mathrm{nmol} / \mathrm{L}\right)$. To our knowledge, osthole may be the most potent natural CFTR activator identified so far. However, the affinity of osthole in potentiating
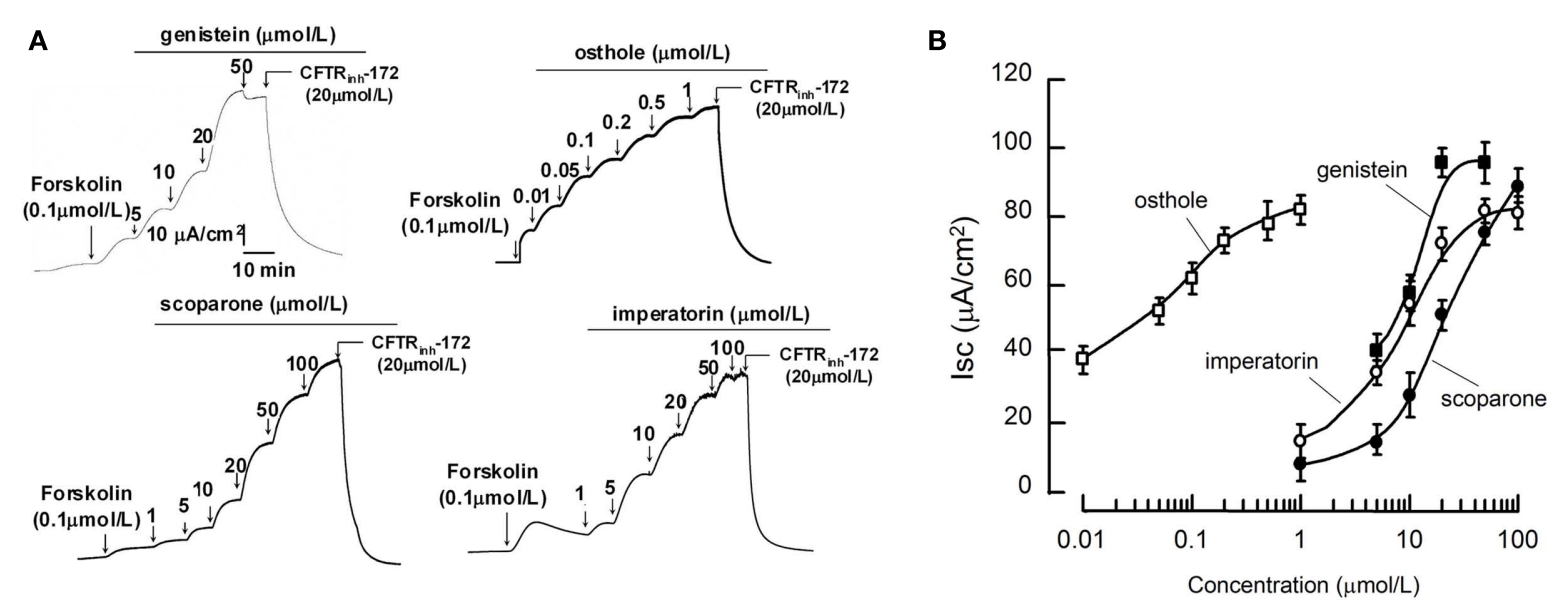

FIGURE 2 | Short-circuit current analysis of the natural coumarins in FRT cells expressing wild-type CFTR. (A) Representative tracings of wt-CFTR-mediated apical membrane $\mathrm{Cl}^{-}$currents are shown. Measurements were performed after the basolateral membrane of Fischer rat thyroid (FRT) cells had been permeabilized with $250 \mathrm{mg} / \mathrm{mL}$ amphotericin $\mathrm{B}$ and in the

presence of a $60-\mathrm{mmol} / \mathrm{L}$ basolateral to apical transepithelial $\mathrm{Cl}^{-}$gradient. Where indicated, various concentrations of the compounds were added to the apical chamber solutions. (B) Dose-response analysis indicates that the natural coumarins stimulated $\mathrm{Cl}^{-}$currents in a concentration-dependent manner. (mean $\pm S E, n=6$ ). 


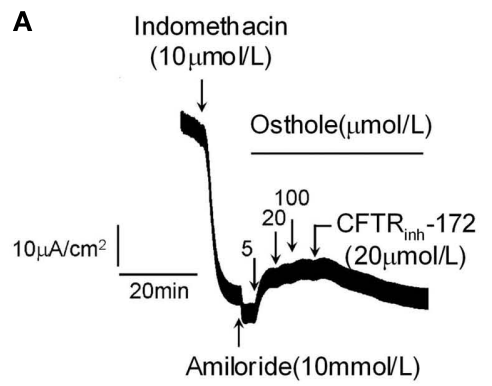

B

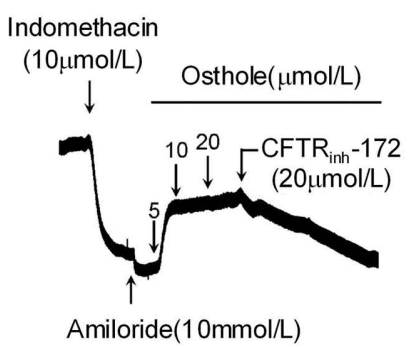

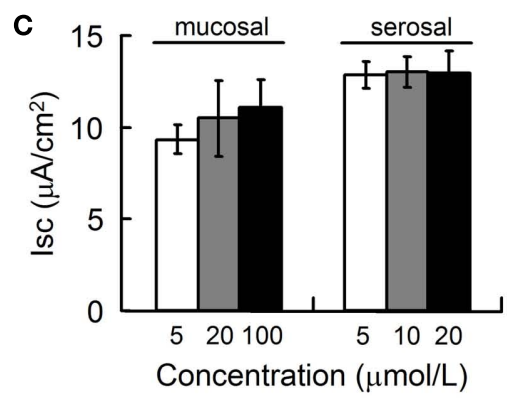

FIGURE 3 | Stimulation of mucosal $\mathrm{Cl}^{-}$current of rat colon by osthole. Osthole-stimulated transepithelial $\mathrm{Cl}^{-}$current was measured after inhibition of $\mathrm{Na}^{+}$current by amiloride $(10 \mu \mathrm{mol} / \mathrm{L})$ and prostaglandin production by indomethacin $(10 \mu \mathrm{mol} / \mathrm{L})$.
Where indicated, osthole and $\mathrm{CFTR}_{\mathrm{inh}}-172$ was added. Osthole was added to mucosal (A) and serosal (B) sides separately. (C) Summary of mucosal $\mathrm{Cl}^{-}$current stimulated by osthole. (mean $\pm \mathrm{SE}, n=6)$.

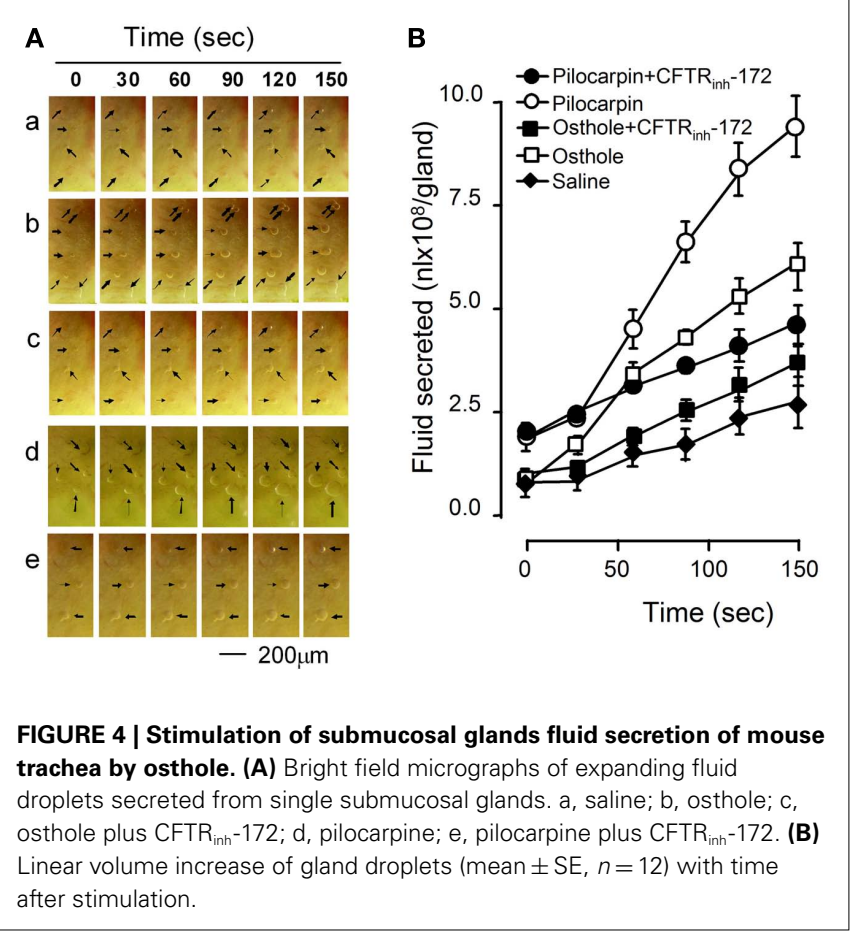

$\Delta$ F508-CFTR $(\sim 10 \mu \mathrm{mol} / \mathrm{L}$; Xu et al., 2008) is $>200$-fold lower than in activating wt-CFTR. The altered gating mechanism of $\triangle$ F508-CFTR may be further investigated using osthole as a probe.

The effect of coumarin compounds on CFTR-mediated fluid secretion in live tissues was demonstrated in two models. In the first model, osthole-stimulated significant $\mathrm{Cl}^{-}$secretion in freshly isolated rat colonic mucosa with maximal effect at $5 \mu \mathrm{mol} / \mathrm{L}$

\section{REFERENCES}

Berdiev, B. K., Qadri, Y. J., and Benos, D. J. (2009). Assessment of the CFTR and $\mathrm{ENaC}$ association. Mol. Biosyst. 5, 123-127.

Boucherot, A., Schreiber, R., and Kunzelmann, K. (2001). Regulation and properties of KCNQ1 (K(V)LQT1) and impact of the cystic fibrosis transmembrane conductance regulator. Membr. Biol. 182, 39-47.

Castro-Parodi, M., Levi, L., Dietrich, V., Zotta, E., and Damiano, A. E. (2009). CFTR may

in Ussing chamber short-circuit current assay. The effect is fully reversed by $\mathrm{CFTR}_{\text {inh }}-172$. In the second model, osthole at $10 \mu \mathrm{mol} / \mathrm{L}$ stimulated $\sim 3$-fold more rapid fluid secretion by tracheal single submucosal gland than that in the control. The stimulated secretions were also fully abolished by CFTR $_{\text {inh }}-172$. These results clearly indicated that coumarin compound osthole can effectively stimulate $\mathrm{CFTR} \mathrm{Cl}^{-}$transport and fluid secretion in live epithelial tissues and the effect is through activation of CFTR chloride channel.

The mechanisms of CFTR activation by coumarin compounds remain unclear. CFTR activation involves several indirect pathways and direct interactions (Hwang and Sheppard, 1999; Schultz et al., 1999). Because coumarins modulate many intracellular pathways (Wu et al., 2009), its activity in activating CFTR may involve both direct and indirect interactions with the CFTR protein. Our previous studies suggested that coumarin compounds may activate wild-type and $\triangle$ F508-CFTR chloride channel activity by a direct interaction with CFTR protein (Ma et al., 2002; Xu et al., 2008), but precise molecular mechanisms need to be fully characterized by systematic electrophysiological studies.

In conclusion, we have demonstrated for the first time that natural coumarin compound osthole can effectively stimulate CFTRmediated $\mathrm{Cl}^{-}$secretion in colonic mucosa and fluid secretion in tracheal submucosal glands. The results suggested the therapeutic potential of natural coumarin compounds for the treatment of CF and other CFTR-related diseases.

\section{ACKNOWLEDGMENTS}

We thank Qingyan Li and Lianhui Dou for their assistance in animal experiments. This work was supported by National Natural Science Fund (No. 30973577), Dalian Municipal Science and Technology Fund (2008E11SF162), and National Basic Research Program of China (973 Program, No. 2009CB521900).

modulate AQP9 functionality in preeclamptic placentas. Placenta 30, 642-648.

Cohn, J. A., Friedman, K. J., Noone, P. G., Knowles, M. R., Silverman, L. M., and Jowell, P. S. (1998). Relation between mutations of the cystic fibrosis gene and idiopathic pancreatitis. N. Engl. J. Med. 339, 653-658.

Galietta, L. V., Jayaraman, S., and Verkman, A. S. (2001). Cell-based assay for high throughput quantitative screening of CFTR chloride 
transport agonists. Am. J. Physiol. Cell Physiol. 281, C1734-C1742.

Greger, R. (2000). Role of CFTR in the colon. Annu. Rev. Physiol. 62, 467-491.

Hanrahan, J. W., and Wioland, M. A. (2004). Revisiting cystic fibrosis transmembrane conductance regulator structure and function. Proc. Am. Thorac. Soc. 1, 17-21.

He, L., Aleksandrov, A. A., Serohijos, A. W., Hegedus, T., Aleksandrov, L. A., Cui, L., Dokholyan, N. V., and Riordan, J. R. (2008). Multiple membrane-cytoplasmic domain contacts in the cystic fibrosis transmembrane conductance regulator (CFTR) mediate regulation of channel gating. J. Biol. Chem. 283, 26383-26390.

He, Q., Zhu, J. X., Xing, Y., Tsang, L. L., Yang, N., Rowlands, D. K., Chung, Y. W., and Chan, H. C. (2005). Tetramethylpyrazine stimulates cystic fibrosis transmembrane conductance regulator-mediated anion secretion in distal colon of rodents. World J. Gastroenterol. 11, 4173-4179.

Ho, K. (1998). The ROMK-cystic fibrosis transmembrane conductance regulator connection: new insights into the relationship between ROMK and cystic fibrosis transmembrane conductance regulator channels. Curr. Opin. Nephrol. Hypertens. 7, 49-58.

Hryciw, D. H., and Guggino, W. B. (2000). Cystic fibrosis transmembrane conductance regulator and the outwardly rectifying chloride channel: a relationship between two chloride channels expressed in epithelial cells. Clin. Exp. Pharmacol. Physiol. 27, 892-895.
Hwang, T. C., and Sheppard, D. N. (1999). Molecular pharmacology of the CFTR Cl- channel. Trends Pharmacol. Sci. 20, 448-453.

Jovov, B., Ismailov, I. I., Berdiev, B. K., Fuller, C. M., Sorscher, E. J., Dedman, J. R., Kaetzel, M. A., and Benos, D. J. (1995). Interaction between cystic fibrosis transmembrane conductance regulator and outwardly rectified chloride channels. J. Biol. Chem. 270, 29194-29200.

Kerem, B., Rommens, J. M., Buchanan, J. A., Markiewicz, D., Cox, T. K., Chakravarti, A., Buchwald, M., and Tsui, L. C. (1989). Identification of the cystic fibrosis gene: genetic analysis. Science 245, 1073-1080.

Kunzelmann, K. (1999). The cystic fibrosis transmembrane conductance regulator and its function in epithelial transport. Rev. Physiol. Biochem. Pharmacol. 137, 1-70.

Levin, M. H., and Verkman, A. S. (2005). CFTR-regulated chloride transport at the ocular surface in living mice measured by potential differences. Invest. Ophthalmol. Vis. Sci. 46, 1428-1434.

Li, H., and Sheppard, D. N. (2009). Therapeutic potential of cystic fibrosis transmembrane conductance regulator (CFTR) inhibitors in polycystic kidney disease. BioDrugs 23, 203-216.

Ma, T., Vetrivel, L., Yang, H., Pedemonte, N., Zegarra-Moran, O., Galietta, L. J., and Verkman, A. S. (2002). High-affinity activators of cystic fibrosis transmembrane conductance regulator (CFTR) chloride conductance identified by highthroughput screening. J. Biol. Chem. $277,37235-37241$.
Machen, T. E., Chandy, G., Wu, M. Grabe, M., and Moore, H. P. (2001). Cystic fibrosis transmembrane conductance regulator and $\mathrm{H}^{+}$permeability in regulation of Golgi $\mathrm{pH}$. JOP 2, 229-236.

Marcet, B., and Boeynaems, J. M. (2006). Relationships between cystic fibrosis transmembrane conductance regulator, extracellular nucleotides and cystic fibrosis. Pharmacol. Ther. 112, 719-732.

Pratha, V. S., Hogan, D. L., Martensson, B. A., Bernard, J., Zhou, R. and Isenberg, J. I. (2000). Identification of transport abnormalities in duodenal mucosa and duodenal enterocytes from patients with cystic fibrosis. Gastroenterology 118 1051-1060.

Riordan, J. R. (2008). CFTR function and prospects for therapy. Annu. Rev. Biochem. 77, 701-726.

Schultz, B. D., Singh, A. K., Devor, D. C., and Bridges, R. J. (1999). Pharmacology of CFTR chloride channel activity. Physiol. Rev. 79, S109-S144.

Song, Y., and Verkman, A. S. (2001). Aquaporin-5 dependent fluid secretion in airway submucosal glands. $J$. Biol. Chem. 276, 41288-41292.

Thiagarajah, J. R., and Verkman, A. S. (2003). CFTR pharmacology and its role in intestinal fluid secretion. Curr. Opin. Pharmacol. 3, 594-599.

Wu, L., Wang, X., Xu, W., Farzaneh, F., and $\mathrm{Xu}$, R. (2009). The structure and pharmacological functions of coumarins and their derivatives. Curr. Med. Chem. 16, 4236-4260.

Xu, L., Na, W., Liu, X., Hou, S., Lin, S., Yang, H., and Ma, T. (2008). Identification of natural coumarin compounds that rescue defective $\triangle$ F508-CFTR chloride channel gating. Clin. Exp. Pharmacol. Physiol. $35,878-883$.

Yang, H., Shelat, A. A., Guy, R. K., Gopinath, V. S., Ma, T., Du, K., Lukacs, G. L., Taddei, A., Folli, C., Pedemonte, N., Galietta, L. J., and Verkman, A. S. (2003). Nanomolar affinity small molecule correctors of defective delta F508-CFTR chloride channel gating. J. Biol. Chem. 278, 35079-35085.

Conflict of Interest Statement: The authors declare that the research was conducted in the absence of any commercial or financial relationships that could be construed as a potential conflict of interest.

Received: 01 March 2011; paper pending published: 23 March 2011; accepted: 06 September 2011; published online: 27 September 2011.

Citation: Yang $H, X u L-N$, Sui $Y$ $j$, Liu $X$, He C-y, Fang R-y, Liu Hao F and Ma T-H (2011) Stimulation of airway and intestinal mucosal secretion by natural coumarin CFTR activators. Front. Pharmacol. 2:52. doi: 10.3389/fphar.2011.00052

This article was submitted to Frontiers in Ethnopharmacology, a specialty of Frontiers in Pharmacology.

Copyright ( 2011 Yang, Xu, Sui, Liu, He, Fang, Liu, Hao and Ma. This is an open-access article subject to a nonexclusive license between the authors and Frontiers Media SA, which permits use, distribution and reproduction in other forums, provided the original authors and source are credited and other Frontiers conditions are complied with. 